\title{
IMPLEMENTING A NEW ALGORITHM FOR ANALYSIS OF PROTOCOL EFFICIENCY USING STABILITY AND DELAY TRADEOFF IN MANET
}

\author{
Shivashankar \\ Dept. of Medical Electronics \\ Dr.Ambedkar Institute of \\ Technology, \\ Bangalore-560 056
}

\begin{abstract}
In this paper, we present an extensive ns-2.33 simulation based performance comparison of four widely known stability-oriented on-demand Mobile Adhoc Network (MANET) routing protocols. Our simulations show that modified Power DSR (PDSR) routes are more stable than existing DSR routes, which are more stable than DSDV and AODV routes. This also results in an increased packet delivery ratio for PDSR in comparison to that of DSR and DSDV. On the other hand, based on the energy consumed per packet and the average energy used per node, DSR is better than DSDV, which is better than PDSR. At low network density and mobility, PDSR routes incur the lowest delay and as the network density and node mobility increases, DSR incurs lower delay. Thus, we see a stability-delay-energy consumption tradeoff among these three stability-oriented routing protocols. Regarding the fairness of node usage, we observe that routes get distributed more evenly with increase in the node mobility and network density. But, still there is an appreciable variation in the energy consumption per node as only the chain of nodes that form stable routes are exhausted to a greater extent. A routing protocol that incurs fewer transitions will lose fewer data packets, create less routing overhead and also maintain in-order data delivery. At the same time, by staying with a long-lived route, the routing protocol may incur a longer end-to-end delay. We intend to explore this tradeoff between stability and delay and try to come with up a metric that would quantify the efficiency of a MANET routing protocol with respect to both stability and delay. On these lines, we introduce the idea of Stability-Delay Tradeoff $(S D T)$ as a measure of the efficiency of a MANET routing protocols.
\end{abstract}

Keywords: PDSR, DSR, DSDV, MANET, packet delivery ratio, stability-oriented, $S D T$.

\section{INTRODUCTION}

Wireless networks are being utilized more and more every day due to the flexibility and convenience they provide. People dislike being tied down, and relish in movement free from hindrance. For instance, someone who is required to move around a lot would most likely choose to invest in a laptop instead of a desktop computer. Much like someone choosing a laptop instead of a desktop computer, people choose to rely on wireless networks and other flexible means of communication.

\author{
Dr.G.Varaprasad \\ Dept. of CSE \\ BMSCE \\ Bangalore-19
}

Daily MANETs, a type of wireless network, are being looked to for an even further flexible means of communication. Unlike cell phones that depend on a central authority for communication (a fixed-location cell phone tower within range), a MANET has no need for a central authority in order to provide a flexible means of communication. With a MANET, each person that is part of the network is in essence like a little mobile cell phone tower. In the modern world such mobility is becoming more and more advantageous, and even essential; for example the use of MANETs can be vital for tactical military operations and disaster relief. Therefore the need for a MANET strategy which uses little energy and is also stable and efficient in its method of communication is an important asset to many industries and organizations. In this paper we will ultimately introduce three strategies aimed at creating stable broadcast topologies based on bottleneck path routing, but also give a general overview of the graph algorithms that are their backbone.

Given the plethora of applications in mobile ad hoc wireless networks, one would like to know the performance of a routing protocol when used in different application scenarios. Specifically, it would be ideal to evaluate the performance of a routing protocol relative to the theoretical optimum computed under the same conditions in which the protocol was run. Using this algorithm, we quantify the efficiency of a MANET routing protocol with respect to path stability by taking the ratio of the actual number of route transitions required by the routing protocol to that of the optimal number of route transitions.

Similarly, using the shortest mobile path algorithm proposed, we quantify the efficiency of a MANET routing protocol with respect to the end-to-end delay. Maximum path stability (i.e., minimum route transitions) and minimum end-to-end delay are not something that can be easily achieved simultaneously. Aiming for minimum end-to-end delay (or for that matter any path metric) can lead to unnecessary route transitions. On the other hand, if we stay with more stable paths, it may lead to longer end-to-end delay, which is not good for time-critical applications.

In a two-dimensional space of stability versus delay, we define the stability-delay tradeoff $(S D T)$ as the proximity of the protocol's actual stability and delay with respect to the optimal 
stability and delay possible in the network under the same conditions. We also suggest methodologies to quantify SDT based on the application's stability and delay requirements. Using the performance metrics obtained under the MERIT framework, we evaluate the SDT of AODV, DSR, DSDV and PDSR. The quantitative measure of the tradeoff can be then used to select the best among the routing protocols that satisfy the application's stability and delay requirements.

The rest of the paper is organized as follows: In Section 2 we study some previous research work on MANET routing protocols and details of stability and delay ratio. In Section 3, we present a design and implementation of stability and delay ratio-tradeoff in MANETs. In Section 4 we introduce the Stability ratio, Delay ratio and Stability-Delay tradeoff as measures of protocol efficiency. And also we present the Stability ratio and Delay ratio obtained for AODV, DSR, DSDV and PDSR under different conditions of offered load and node mobility in the same chapter. In Section 5, we calculate the SDT based on the results and rank the four MANET routing protocols for diverse simulation conditions. Section 6 concludes the protocol affiance with respect to all four protocols.

\section{SOME OF THE EXISTING RESEARCH WORK}

A significant number of research efforts have been devoted to investigate MANETs over the past few years [1, 2, 3]. Interest in MANETs is due to their promising ubiquitous connectivity beyond that is currently being provided by the Internet. Firstly, MANETs are easily deployed allowing a plug-and-communicate method of networking. Secondly, MANETs need no infrastructure [4]. Eliminating the need for an infrastructure reduces the cost for establishing the network. Moreover, such networks can be useful in disaster recovery where there is not enough time or resources to install and config. an infrastructure. Thirdly, MANETs also do not need central management. Hence, they are used in military operations where units are moving around the battle field and a central unit cannot be used for synchronization [5]. Nodes forming and Ad Hoc network are required to have the ability to double up as a client, a server, and a router simultaneously [6]. Moreover, these nodes should also have the ability to connect to and automatically config. [7] to start transmitting data over the network. It is impractical to expect a MANET to be fully connected, where a node can directly communicate with every other node in the network. Typically, nodes are obliged to use a multi-hop path for transmission [8], and a packet may pass through multiple nodes before being delivered to its intended destination. A number of MANET routing protocols were proposed in the last decade. These protocols [9] can be classified according to the "routing strategy" that they follow to find a path "route" to the destination. These protocols perform variously depending on type of traffic, number of nodes, rate of mobility, etc...

\section{METHODOLOGIES AND PROPOSED ALGORITHM}

\subsection{Basic Algorithm}

Let the sequence of network topology changes be represented by the graph sequence $G_{I} G_{2} G_{3}$ as shown in Fig. 1. The sourcedestination pair is 1 to 6 . The link weights in these graphs represent the link delays. The sequence of graphs is constructed at the instants when the optimal delay path breaks. The least delay mobile path is the sequence of optimal delay paths. Running Dijkstra's algorithm on $G_{1}, G_{2}$ and $G_{3}$ would yield the optimal delay paths mentioned below the corresponding graphs. Also note that there is a common path 1-3-5-6 in all the three graphs. This is the stable mobile path.

In Fig 1, the least delay mobile path is [(1-2-4-6), (1-3-4-6), $(1-2-4-6)]$ and its weight is $w_{1}(1-2-4-6)+w_{2}(1-3-4-6)+$ $w_{3}(1-2-4-6)=5+6+6=17$. The number of route transitions is 2 . On the other hand, if we had used the stable mobile path 13-5-6 throughout the 1-6 session, the total end-to-end delay incurred would be $w_{1}(1-3-5-6)+w_{2}(1-3-5-6)+w_{3}(1-3-5-6)$ $=7+8+10=25$.

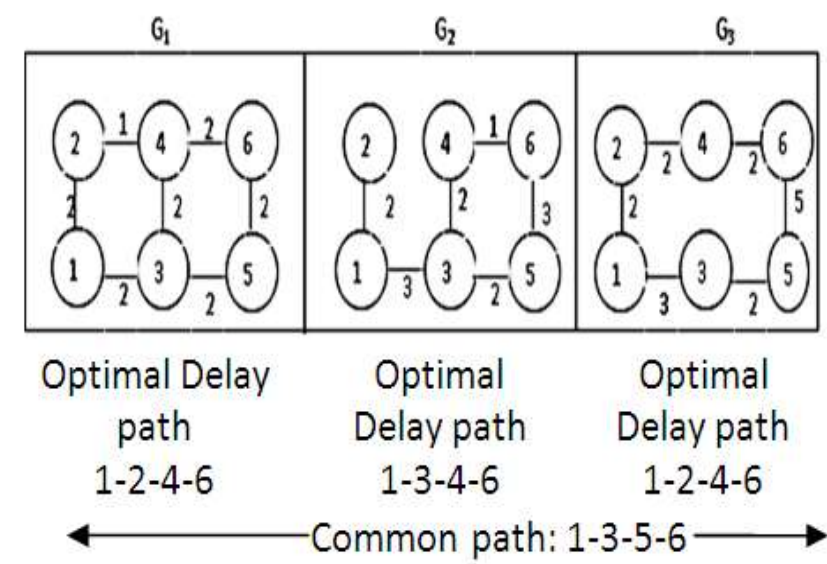

Fig 1: llustrate Stability Delay Tradeoff

The number of route transitions is 0 . This simple example shows that the delay incurred by a stable mobile path can be appreciably larger than that of a least delay mobile path; on the other hand at least delay mobile path may have larger number of route transitions than that in a stable mobile path. The example also shows that the least delay mobile path and stable mobile path can sometimes have no paths in common between them.

\subsection{Measures of Routing Protocol Efficiency}

\subsubsection{Stability Ratio}

For a given source-destination $s$ - $d$ session, let trans $_{\text {actual }}$ be the actual number of route transitions made by a routing protocol $R$ and let trans $_{\text {opt }}$ be the minimum for the number of route transitions required for the same $s-d$ session. The Stability ratio 
captures the impact of the offered load and the routing policies of the protocol on the network topology changes.

$$
\text { Stability Ratio }=\mathrm{E}\left[\frac{\text { trans }_{\text {actual }}+1}{\text { trans }_{\text {opt }}+1}\right]
$$

Note that instead of directly taking the ratios of the actual to the optimal number of transitions, we add 1 to both of them and then take the ratio. It avoids the possibility of the Stability ratio becoming undefined if either the optimal number of transitions is 0 or both the actual and optimal transitions are 0 .

DSR [10] and DSDV [11] use one path at a time, but are capable of maintaining multiple paths to the destination. For example, a source $s$ running either of these two protocols may maintain two paths $P 1$ and $P 2$ to the destination $d$ and it may switch repeatedly between $P 1$ and $P 2$. We do not consider such route switches while computing trans $s_{\text {actual }}$. We only consider route transitions that occur when the source $s$ is not aware of any $s$ - $d$ path at the instant of packet origination and initiates a route discovery to destination $d$. Thus, the Stability ratio also captures the fact that the routing protocol $R$ has used at least trans $_{\text {actual }}+1$ path, while it should have ideally used only trans $_{\text {opt }}+1$ path.

\subsubsection{Delay Ratio}

The least delay mobile path is defined as the mobile path with the minimum end-to-end delay theoretically possible. By sampling the network topology at instants of packet origination and using the link delays (obtained from the simulation trace of the routing protocol) as link weights in the static graphs, the mobile graph $G_{M}=G_{l} G_{2} \ldots G_{T}$ for an $s$ - $d$ session can be generated over the timescale $T$. The least delay mobile path in $G_{M}$ can be then efficiently computed using equations (1) and (2). The actual mobile path is defined as the sequence of routes used by the routing protocol over the timescale $T$. The transition cost between paths is implicitly fixed using the routing trace generated.

Let $P_{\text {ideal }}$ be the least delay mobile path and the end-to-end delay per packet in the least mobile path be denoted $w\left(P_{\text {ideal }}\right)$. Similarly, let $P_{\text {actual }}$ be the actual mobile path of the routing protocol and the end-to-end delay per packet incurred by the routing protocol be denoted as $w\left(P_{\text {actual }}\right)$. The delay ratio is the expected value of the ratios of the end to end delay per packet in the actual mobile path to that in the least delay mobile path. In other words,

$$
\text { Delay Ratio }=\mathrm{E}\left[\frac{W\left(P_{\text {actual }}\right)}{W\left(P_{\text {ideal }}\right)}\right]
$$

\subsubsection{Stability - Delay Tradeoff}

Based on the above discussions, we can say that an ideal situation for any type of application is to use routes that can simultaneously achieve both optimal end-to-end delay and optimal number of route transitions. In other words, when we

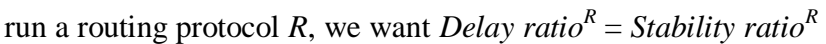
$=1$. In practical terms, if we draw a plot of Stability ratio vs. Delay ratio, we want the routing protocol $R$ 's data point

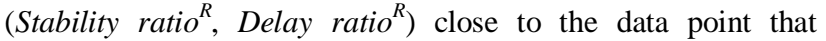
represents the ideal situation, $(1,1)$. The proximity of (Stability ratio $^{R}$, Delay ratio $\left.^{R}\right)$ to $(1,1)$ indicates how close is the routing protocol in achieving minimum end-to-end delay and minimum route transitions at the same time. We refer to this proximity as $S D T$. We propose the $S D T$ as a measure of the efficiency of a MANET routing protocol with respect to both stability and delay.

\section{S-D TRADEOFF BASIC METHODS}

\subsection{Distance-based Method}

Let $R$ be the routing protocol under evaluation. Its Stability ratio $^{R}$ and Delay ratio $^{R}$ are determined using the algorithms discussed in the previous sections. Let us introduce two new variables $S^{R}{ }_{\text {dist }}$ and $D^{R}$ dist which are respectively one less than

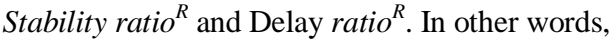

$$
\begin{aligned}
S_{{ }_{\text {dist }}} & =\text { Stability }_{\text {ratio }^{R}-1} \\
D_{\text {dist }^{R}} & =\text { Delay ratio }
\end{aligned}
$$

In a two-dimensional plot of Stability ratio Vs Delay ratio ${ }^{R}, S^{R}$ dist basically indicates how far is Stability ratio $^{R}$ from the ideal value of 1. Similarly, $D^{R}$ dist indicates how far is Delay ratio $^{R}$ from the ideal value of 1 . A direct distance-based estimate of the $S D T$ of protocol $R$ can be simply given by the Euclidean distance between points $(1,1)$ and (Stability ratio ${ }^{R}$, Delay ratio $^{R}$ ). In other words,

$$
S D T_{\text {dist }}^{R}=\sqrt{\left(S_{\text {dist }}^{R}\right)^{2}+\left(D_{\text {dist }}^{R}\right)^{2}}
$$

\subsection{Area-based Method}

When both stability and delay are to be given equal importance, we would basically want both the Stability and Delay ratios to be as close as possible to $(1,1)$ and a unit increase or decrease in the Stability ratio to result in a similar change in the Delay ratio and vice-versa. We capture this by projecting the line joining (1, 1) and (Stability ratio ${ }^{R}$, Delay ratio $^{R}$ ) to the $45^{\circ}$ line through the ideal point $(1,1)$. In other words, we are basically transforming the rectangle of width $D^{R}$ dist and height $S^{R}{ }_{\text {dist }}$ to a square of side representing the stability - delay tradeoff, $S D T_{\text {area }}^{R}$. Therefore,

$$
S D T^{R}{ }_{\text {area }}=\sqrt{S_{\text {dist }}^{R} * D_{\text {dist }}^{R}}
$$

\subsection{Distance - Weight based Method}

Different applications have different stability and delay requirements. There might be real-time applications like VoIP that want the end-to-end delay to be as close as possible to the theoretical optimum and are not at all concerned about stability. 
Similarly, resource-constrained environments like sensor networks may want to save resources by undergoing limited number of route transitions, preferably close to the theoretical optimum and are not at all concerned about the delay incurred. We handle the above complications, by allowing the application user to specify the importance given to stability and delay using weights $W_{\text {Stability }}$ and $W_{\text {Delay }}$ respectively, such that $W_{\text {Stability }}+$ $W_{\text {Delay }}=1$. We now define the (distance, weight)-based quantitative estimate of the $S D T$ for a routing protocol $R$ as

$$
S D T_{\text {dist }, W}^{R}=\sqrt{W_{\text {Stability }}\left(S_{\text {dist }}^{R}\right)^{2}+W_{\text {Delay }}\left(D_{\text {dist }}^{R}\right)^{2}}
$$

When $W_{\text {Stability }}$ and $W_{\text {Delay }}=0.5, S D T_{\text {area }}^{R} \leq S D T_{\text {dist }, W}^{R} \leq S D T_{\text {dist }}^{R}$

The efficiency of a routing protocol is inversely related to its $S D T$. In other words, smaller the SDT value, larger the efficiency of the routing protocol and vice-versa.

\section{SIMULATION SETUP AND RESULT FOR STABILITY AND DELAY RATIO}

Extensive simulations were conducted using NS-2.33. While the implementation of DSR and AODV routing protocols is provided by [12], however, PDSR implementation is provided by [13]. The simulated network consisted of 100 nodes randomly scattered in a $2000 \times 2000 \mathrm{~m}$ area at the beginning of the simulation. The tool setdest [14] was used to produce mobility scenarios, where nodes are moving at six different uniform speeds ranging between 0 to $15 \mathrm{~m} / \mathrm{s}$ with a margin of \pm 1 and a uniform pause time of 10s. We simulated the steady-state conditions of the network with three types of traffic models; namely PDSR, Pareto and Exponential. These were generated using the tool pdsrgen.tcl [14], with the following parameters:

Table1.1: Simulation parameters list

\begin{tabular}{|l|l|}
\hline Network Simulator & NS-2.33 Version \\
\hline Network Size & $2000 x 2000$ \\
\hline Number of Mobile Nodes & 100 \\
\hline Signal Processing Model & Two - ray ground \\
\hline Transmission range & $250 \mathrm{~m}$ \\
\hline MAC layer & IEEE-802.11G \\
\hline Link bandwidth & 2Mbps \\
\hline Routing Protocols & DSR,AODV, DSDV and PDSR \\
\hline Mobility Model & Random way \\
\hline Maximum Node Speed & $1,5,10$ and $15 \mathrm{~m} / \mathrm{s}$ \\
\hline Traffic Model & CBR,UDP \\
\hline Data Packet size & 128 bytes \\
\hline
\end{tabular}

We used the $n s-2.33$ simulator to run the AODV, DSR, DSDV and PDSR routing protocols and collect their routing trace and the network's mobility history. Our simulation environment and the simulation models are summarized in Table 4.1. We present the results for the Stability ratio and the Delay ratio in this section and then use them to evaluate the SDT. For fairness, we used identical mobility and traffic scenarios to evaluate the four protocols. Each data point in Fig.s 2 and 3 represents the average value of the ratios of all the $15 s-d$ sessions in 5 runs of each of the protocol for the same traffic model, but different randomly generated mobility scenarios. The performance of the protocols at low load (1 Packet / sec) and at high load (4 Packets / sec) is presented in Fig.s 2 and 3 respectively.

\subsection{Delay Ratio at Low Load}

As the speed increases in fig. 2, all the four protocols show an increase in the Delay ratio. All the four protocols have Delay ratios somewhat closer to each other. At low mobility, DSDV has a Delay ratio lower than that of AODV, but as the speed increases the Delay ratio of DSDV becomes larger than that of the other three protocols. The Delay ratios of AODV, DSR are not far different from each other; nevertheless DSR has the lowest Delay ratio irrespective of the speeds. Also to be noted is that the Delay ratio of AODV and DSR slowly increase with increase in speed, where as the Delay ratio of DSDV and PDSR increases relatively faster with increase in speed.

\subsection{Delay Ratio at High Load}

Irrespective of mobility, all the four protocols incur a higher Delay ratio than that at low load (in fig. 3). A more interesting observation is that the Delay ratios of all the four protocols decrease with increase in speed, contrary to what is observed at low load conditions. Delay ratio is high at low velocities, indicating the need to circumvent around heavily loaded nodes.

As the node velocity increases, the effect of better route distribution offsets the route discovery latency, eventually decreasing the Delay ratio. Also, at high packet rates, nodes become aware of more and better routes to the destination and this is very much evident at high speeds. At low mobility, DSR has the lowest Delay ratio of all the four protocols, but as the mobility increases, AODV becomes the best. DSDV Delay ratios are larger and far away from those of AODV and DSR. The Delay ratio of PDSR is less than that of AODV at low mobility. Longer hop count paths is offset by less routing overhead in PDSR. This could be attributed to the stability of the routes chosen. The Delay ratios of AODV and DSR are close to each other at high mobility conditions. 


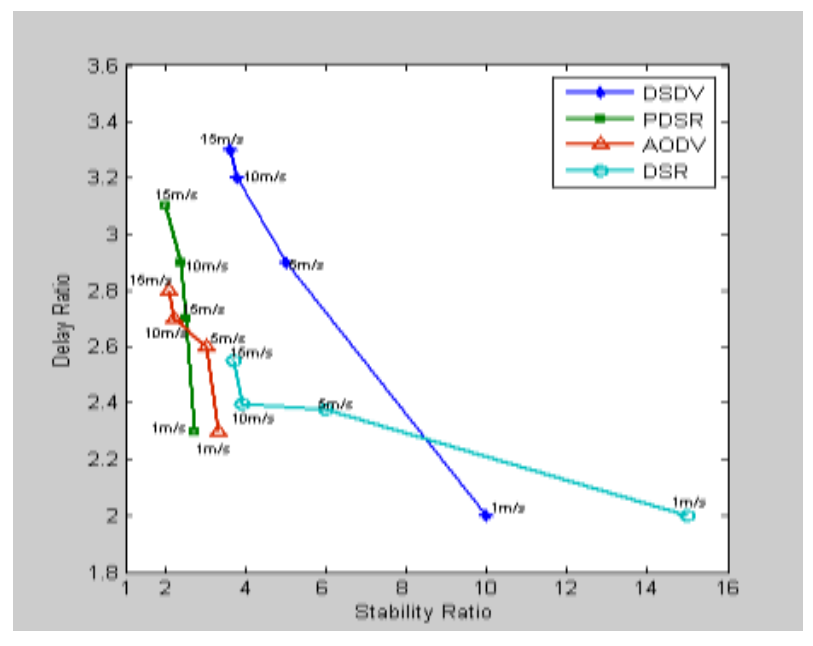

Fig.2. Stability Ratio Vs Delay Ratio ( load: 1 packet / sec)

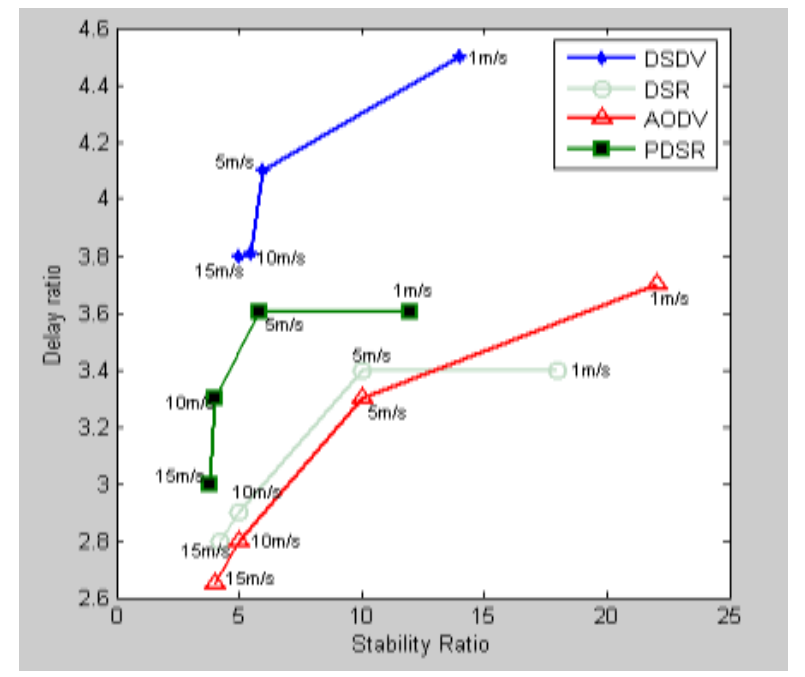

Fig.3. Stability Ratio Vs Delay Ratio ( load: 8 packets / sec)

\subsection{Stability Ratio at Low Load}

Even though the absolute number of route transitions increases in fig. 2 with node velocity, the Stability ratio decreases with increase in velocity. This is due to the pseudo link failures perceived because of MAC contention and HELLO packet losses. PDSR has the lowest Stability ratio of all the four at all speeds; as the speed increases, AODV catches up with PDSR. In AODV, the source maintains only one route to the destination and sticks on to that as long as it exists. At low mobility conditions, DSR and DSDV have high Stability ratios, indicating these protocols change paths unnecessarily when the network is close to static. But as the mobility increases, the Stability ratios of all the four protocols start to decrease and converge close to each other.

\subsection{Stability Ratio at High Load}

At high load in fig. 3, all the four protocols incur higher Stability ratios than those at low loads. This could be attributed to the possibility of congestion at high data loads. PDSR has the lowest Stability ratio at all speeds. At low mobility, DSR has the highest Stability ratio. Similar to the behavior observed in low load conditions, as mobility increases, the Stability ratios of all the four protocols decrease and converge close to each other.

\subsection{SDT Calculations - Ranking of MANET Routing Protocols}

Fig.s 4 and 5 present the Stability-Delay Tradeoff values for the Stability Ratio and Delay Ratio results presented in fig.s 2 and 3. The $S D T$ values presented here represent the situation when stability and delay are to be given equal importance, i.e.,

$W_{\text {stability }}=W_{\text {Delay }}=0.5$.

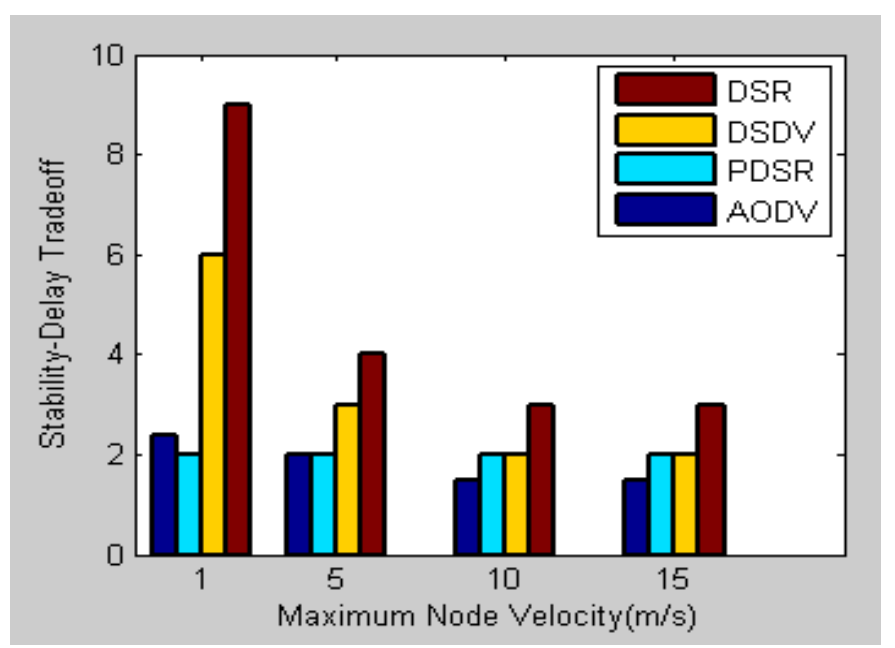

Fig.4(a). Distance-Weight Method (Load:1 Packet/sec)

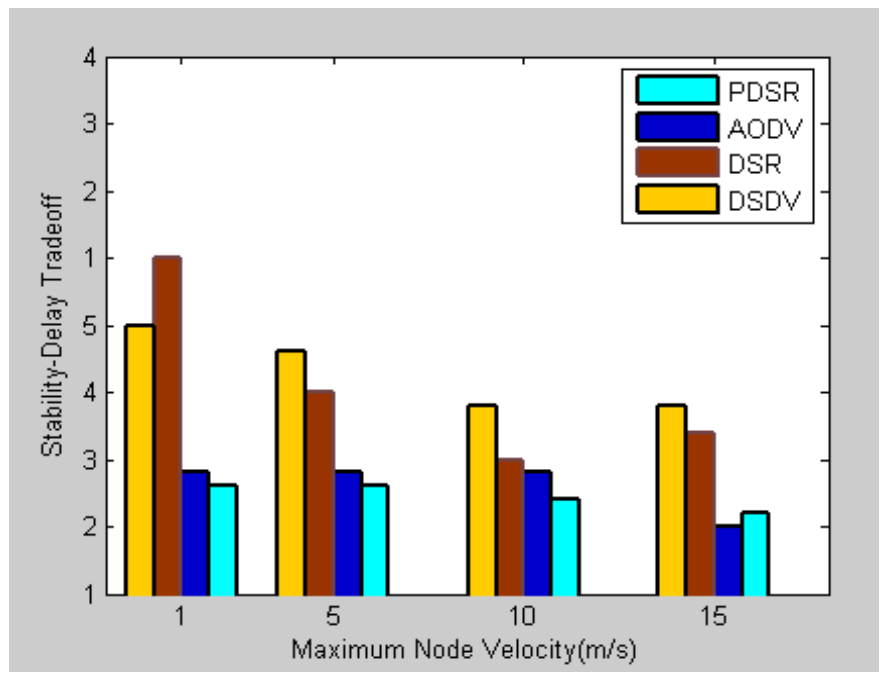

Fig.4(b). Area Based Method (Load: 1 Packet/sec) 
The SDT values obtained from the distance - weight based method (in fig.s 4(a) and 5(a)) are at most a factor of 3 times larger than that obtained using the area-based method (in fig.s 4(b) and 5(b). The results also show that the ranking of the protocols using the two methods need not be always similar.

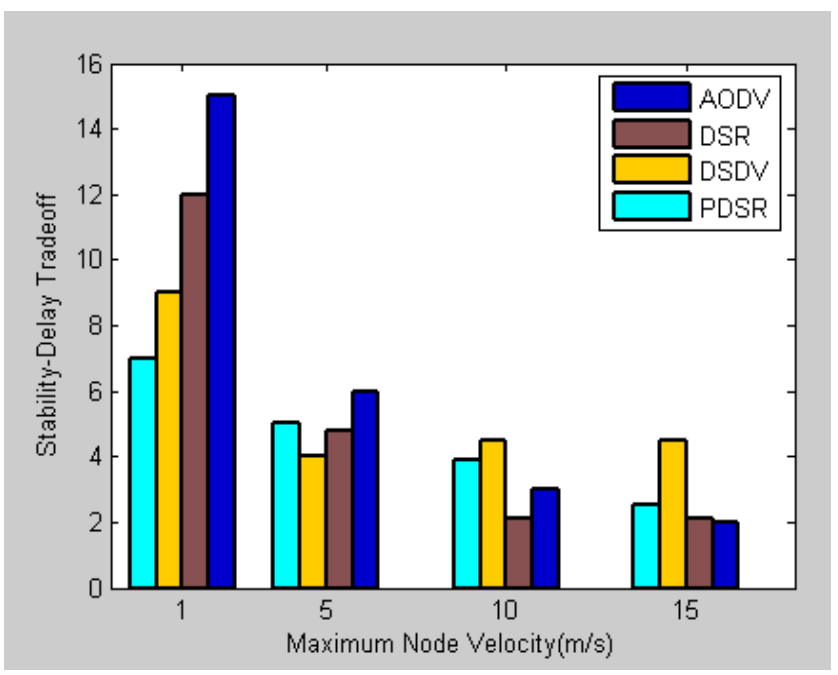

Fig.5(a). Distance- Weight Method Fig. (Load: 8 Packets/sec)

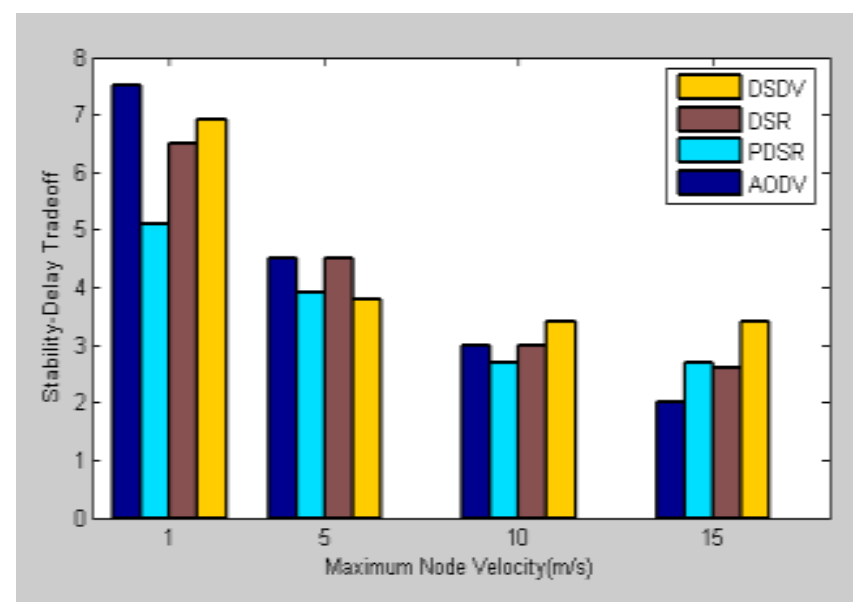

5(b) Area Based Method (Load: 8 Packets/sec)

For example, in the case of $1 \mathrm{~m} / \mathrm{s}$ and 8 packets / sec, DSDV is clearly better than DSR using the distance-weight method, where as DSR is slightly better than DSDV using the area-based method. In general, if equal importance is to be given to stability and delay, AODV and PDSR seem to be the best protocols at 1 packet / sec; whereas at 8 packets / sec, PDSR is best at low velocities, and AODV catches up with PDSR as the velocity increases.

\section{CONCLUSION}

We could draw two significant conclusions. One, we see a stability-delay-energy consumption tradeoff among the stabilityoriented routing protocols for MANETs. Higher the stability, higher is the end-to-end delay and more likely higher energy consumption per packet. RPDSR reduces this tradeoff to a certain extent by maintaining a proper balance between the route propagation load and route stability. The results presented the illustrate tradeoff between stability and delay for MANET routing protocols. Protocols designed to give stable routes yield a larger delay, while protocols designed to select minimum hop / delay routes are less stable. PDSR is the best protocol in terms of the SDT. An interesting observation in fig.s 4 and 4 is that as the node mobility is increased, the SDT of the different routing protocols converges to a constant. This indicates that the route selection maintenance strategies of the protocols impact their performance only at low to moderate node mobility and as the node mobility gets high, the route maintenance procedures become fruitless and all the routes are discovered using flooding of control packets. The lifetime of the routes chosen by the protocols converges as the node mobility increases: PDSR's strategy of discovering stable routes using the past history does not help much in choosing routes with longer lifetime.

\section{REFERENCES}

[1] S. R. Das, C. E. Perkins, E. M. Royer and M. K. Marina, "Performance Comparison of Two Ondemand Routing Protocols for Ad Hoc Networks," in IEEE Personal Communications Magazine, special issue on Mobile Ad Hoc Networks, Vol. 8, No. 1, pp. 16-29, Feb 2001

[2] MANET Simulation and Implementation at the University of Murcia, Available from http://masimum.dif.um.es/, 2006.

[3] S. Kurkowski, T. Camp, and M. Colagrosso, "MANET Simulation Studies: The Incredibles," in ACM's Mobile Computing and Communications Review, vol. 9, no. 4, pp. 50-61, October 2005

[4] Thomas D. Dyer, Rajendra V. Boppana, "A comparison of TCP performance over three routing protocols for mobile ad hoc networks," MobiHoc 2001: 56-66, 2001

[5] MohammadReza EffatParvar, Naser Yazdani, Farshad Lahooti, Mehdi EffatParvar, "Link Stability Approach and Scalability Method on ODMRP in Ad Hoc Networks" Seventh Annual Communication Networks and Services Research Conference, CNSRC 2009, pp.416-421.

[6] Hao $\mathrm{Xu}$, Dejun $\mathrm{Mu}$, "A Cluster Based Stable Multicast Routing Protocol in Ad Hoc Network", IEEE/IFIP International Conference on Embedded and Ubiquitous Computing, EUC 2008, Vol. 2, pp.723-728. 
[7] Rajashekhar Biradar, Sunilkumar Manvi, Myalara Reddy, "Mesh based multicast routing protocol in mobile ad hoc networks", Proceedings of National Conference on Computer Networks, NCCN 2009, Bangalore, pp.17-22.

[8] I. Stepanov, D. Herrscher, K. Rothermel, "On the impact of radio propagation models on MANET simulation results", Proceedings of 7th International Conference on Mobile and Wireless Communications Networks (MWCN 2005), Marrakech, Morocco, September 2005.

[9] Qing Dai, Jie Wu, "Computation of minimal uniform transmission range in ad hoc wireless networks", Cluster Computing, Springer Science, Vol.8, 2005, pp.127-133.

[10] Harleen Dhillon, Hung Q. Ngo, "CQMP: A meshbased multicast routing protocol with consolidated query packets", IEEE Communications Society /WCNC 2005, pp. 2168-2174.

[11] Y.-C. Tseng, Y.-F. Li, and Y.-C. Chang, "On Route Lifetime in Multihop Mobile Ad Hoc Networks," IEEE Transactions on Mobile Computing, Vol. 2, No. 4, pp. 366-376, Oct.-Dec. 2003.

[12] CMU Monarch Group, "CMU Monarch extensions to the NS-2 simulator." Available fromhttp://www.monarch.cs.cmu.edu/cmu-ns.html, 2006.

[13] The Network Simulator, NS-2, Available fromwww.isi.edu/nsnam/ns, 2006

[14] T. Clausen, and P. Jacquet, "Optimized Link State Routing Protocol (OLSR)". IETF RFC3626, Available from http://menetou.inria.fr/olsr/rfc3626.txt, October 2003.

[15] R. Groenevelt, E. Altman, and P. Nain, "Relaying in Mobile Ad Hoc Networks: The Brownian Motion Mobility Model," Wireless Networks (WINET), Vol. 12, pp. 561-571, May 2006.

[16] T. Spyropoulos, K. Psounis, and C. S. Raghavendra, "Performance Analysis of Mobilityassisted Routing," ACM MobiHoc, Florence, Italy, pp. 49-60, May 2006.
[17] H. AhleHagh, and W. R. Michalson, "Statistical Characteristics of Wireless Network Traffic and Its Impact on Ad Hoc Network Performance," in Advanced simulation Technologies Conference, Orlando, USA, pp. 66-71, April 2003.

[18] J. Borch, D. A. Maltz, D. B. Jognson, Y. Hu, and J. Jetcheva, "A Performance Comparison of Multi-Hop Wireless Ad Hoc Network Routing Protocols," in Proceedings of the 4th ACM/IEEE international conference on Mobile computing and networking, Dallas, Texas, USA, pp. 85-97, 1998.

[19] T. Camp, J. Boleng, B. Williams, L. Wilcox, and W. Navidi, "Performance Comparison of Two Location Based Routing Protocols for Ad Hoc Networks," in Proceedings of the IEEE INFOCOM 2002, The $21^{\text {st }}$ Annual Joint Conference of the IEEE Computer and communications Societies, New York, USA, June 2002.

[20] S. Lee, J. Hsu, R. Hayashida, M. Gerla, and R. Bagrodia, "Selecting a Routing Strategy for Your Ad Hoc Network," Computer Communications, special issue on Advances in Computer Communications and Networks: Algorithms and Applications, vol. 26, no. 7, pp. 723-733, May 2003.

[21] P. Jacquet, P. Muhlethaler, T. Clausen, A. Laouiti, A. Qayyum, and L. Viennot, "Optimized Link State Routing Protocol for Ad-Hoc Networks," in Proceedings of the 5th IEEE Multi Topic Conference (INMIC 2001), 2001.

[22] C. Perkins, E. Belding-Royer, and S. Das, "Ad Hoc On- Demand Distance Vector (AODV) Routing". IETF RFC3561, Available from http://www.ietf.org/rfc/rfc3561.txt?number=3561, July 2003.

[23] David B. Johnson, David A. Maltz, and Josh Broch, "DSR: The Dynamic Source Routing Protocol for Multi-Hop Wireless Ad Hoc Networks," in Ad Hoc Networking, edited by Charles E. Perkins, Chapter 5, pp. 139-172, Addison-Wesley, 2001. 